\title{
Mapping surface residues of eIF5A that are important for binding to the ribosome using alanine scanning mutagenesis
}

\author{
Natália M. Barbosa ${ }^{1}$ Paulo E. G. Boldrin ${ }^{1} \cdot$ Danuza Rossi $^{1} \cdot$ Priscila A. Yamamoto $^{1}$ \\ Tatiana F. Watanabe ${ }^{1} \cdot$ Vitor H. Serrão $^{2} \cdot$ John W. B. Hershey ${ }^{3}$. \\ Christopher S. Fraser ${ }^{3}$ Sandro R. Valentini ${ }^{1}$ Cleslei F. Zanelli ${ }^{1}$
}

Received: 17 February 2016 / Accepted: 11 June 2016 / Published online: 7 July 2016

(C) Springer-Verlag Wien 2016

\begin{abstract}
The translation elongation factor eIF5A is conserved through evolution and is necessary to rescue the ribosome during translation elongation of polyprolinecontaining proteins. Although the site of eIF5A binding to the ribosome is known, no systematic analysis has been performed so far to determine the important residues on the surface of eIF5A required for ribosome binding. In this study, we used clustered charged-to-alanine mutagenesis and structural modeling to address this question. We generated four new mutants of yeast eIF5A: tif51A-4, tif51A-6, tif51A-7 and tif51A-11, and complementation analysis revealed that tif51A-4 and tif51A-7 could not sustain cell growth in a strain lacking wild-type eIF5A. Moreover, the allele tif51A-4 also displayed negative dominance over wild-type eIF5A. Both in vivo GST-pulldowns and in vitro fluorescence anisotropy demonstrated that eIF5A from mutant tif51A-7 exhibited an importantly reduced affinity for the ribosome, implicating the charged residues in cluster 7 as determinant features on the eIF5A surface
\end{abstract}

Handling Editor: E. Agostinelli.

Electronic supplementary material The online version of this article (doi:10.1007/s00726-016-2279-z) contains supplementary material, which is available to authorized users.

Cleslei F. Zanelli

zanellicf@fcfar.unesp.br

1 Department of Biological Sciences, School of Pharmaceutical Sciences, São Paulo State University-UNESP, Rod Araraquara-Jaú Km01, Araraquara, SP 14800-903, Brazil

2 Physics and Interdisciplinary Science Department, Physics Institute of Sao Carlos, University of Sao Paulo-USP, Sao Carlos, SP 13563-120, Brazil

3 Molecular and Cellular Biology Department, University of California, Davis, CA 95616, USA for contacting the ribosome. Notably, modified eIF5A from mutant tif51A-4, despite exhibiting the most severe growth phenotype, did not abolish ribosome interactions as with mutant tif51A-7. Taking into account the modeling eIF5A $+80 \mathrm{~S}+$ P-tRNA complex, our data suggest that interactions of eIF5A with ribosomal protein L1 are more important to stabilize the interaction with the ribosome as a whole than the contacts with P-tRNA. Finally, the ability of eIF5A from tif51A-4 to bind to the ribosome while potentially blocking physical interaction with P-tRNA could explain its dominant negative phenotype.

Keywords Translation elongation · Hypusine - eIF5A · Ribosome binding
Abbreviations
eIF5A Eukaryotic translation initiation factor 5A
TIF51A Gene encoding eIF5A in yeast
EF-P Elongation factor $\mathrm{P}$

\section{Introduction}

The main aspects of protein synthesis are highly conserved throughout evolution, and many steps and factors involved in translation are very similar across all kingdoms of life. Concerning the translation factors, the elongation step of translation is most conserved (Dever and Green 2012; Rodnina and Wintermeyer 2009), and the homologous factors bacterial EF-P and archaeal/eukaryotic eIF5A are examples of such conservation (Rossi et al. 2014).

The former eukaryotic translation initiation factor $5 \mathrm{~A}$ (eIF5A) is an essential translation factor that was originally purified from ribosomal salt wash of rabbit reticulocytes and initially classified as an initiation factor because of its 
ability to stimulate methionyl-puromycin synthesis (Kemper et al. 1976; Benne and Hershey 1978). Notably, elongation factor P (EF-P) is not essential in some bacteria, such as Escherichia coli, but was also initially identified as a stimulatory factor for methionyl-puromycin synthesis in an in vitro reconstituted translation assay (Glick and Ganoza 1975, 1976). Although both eIF5A and EF-P have been previously related to different aspects of protein synthesis and other cellular functions, both have been demonstrated to be necessary to rescue the ribosome during the translation elongation of polyproline-containing proteins (Rossi et al. 2014).

eIF5A is an approximately $15-17 \mathrm{kDa}$ protein (depending on the species) composed of two predominantly $\beta$-barrel domains. The $\mathrm{N}$-terminal domain is SH3-like, predominantly positively charged and more conserved than the rest of the protein. The C-terminal domain consists of an $\mathrm{OB}$ fold found in nucleic acid-binding proteins and is much more negatively charged (Kim et al. 1998). Notably, EF-P contains three domains, instead of two, which gives it a molecular mass of approximately $21 \mathrm{kDa}$. Domains I and II of EF-P contain the same folds as the N-terminal and C-terminal domains of eIF5A, and domain III of EF-P appears to be a duplication of domain II.

An interesting structural aspect of both eIF5A and EF-P is the unique posttranslational modifications they undergo that are necessary for their functions during the elongation of polyproline proteins. These modifications occur on a specific lysine (in all eIF5A and in EF-P of some species) or arginine (in EF-P of other species) that reside at the tip of the N-terminal long loop (Lassak et al. 2015). The site of the modification in eIF5A, called hypusination, is shown in Fig. 1.

In eIF5A, this essential modification comprises the conversion of a lysine to a hypusine and is essential for all archaea and eukaryotes tested so far (Rossi et al. 2014). Hypusine formation occurs in two steps: first, the enzyme deoxyhypusine synthase (Dys1 in Saccharomyces cerevisiae) transfers a 4-aminobutyl moiety from the polyamine spermidine to the $\varepsilon$-amino group of the specific lysine side chain to form deoxyhypusine; then, the second carbon of the deoxyhypusine residue is hydroxylated by deoxyhypusine hydroxylase (Lia1 in S. cerevisiae).

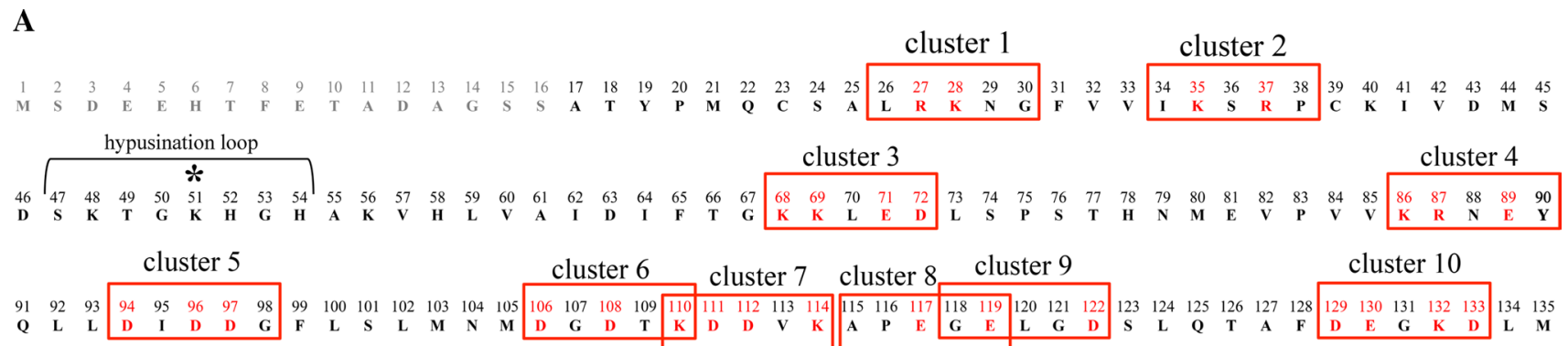

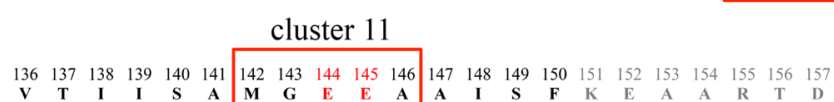

B
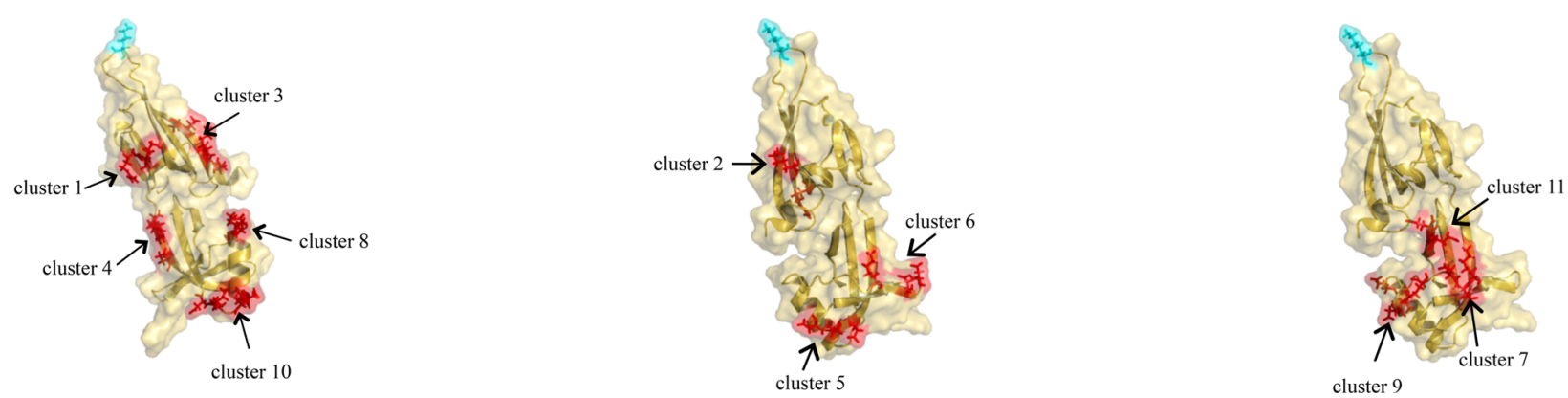

Fig. 1 Residues substituted in charge clustered-to-alanine mutations. a Primary sequence of eIF5A highlighting the mutated clusters, hypusination loop and Lys51, where the modification occurs. The residues not present in the eIF5A crystal structure are in gray. The red boxes indicate the clusters of five amino acid residues with at least two

charged amino acids chosen as targets for the alanine scanning strategy. b eIF5A structure (PDB 3ER0) showing the amino acid residues mutated, highlighted in red and the hypusination site highlighted in cyan (Gentz et al. 2009) 
Although all archaea and eukaryotes undergo hypusination of eIF5A, modifications on EF-P vary depending on the species. The co-occurrence of EF-P containing lysine or arginine and the corresponding modification pathways (described below) are present only in approximately $35 \%$ of the known bacterial genomes, suggesting that either modification of EF-P in other species is not necessary, or there are other types of EF-P modifications yet to be discovered (Lassak et al. 2015). So far, three different posttranslational modifications have been described for EF-P: $\beta$-lysinylation of EF-P containing a lysine at the tip of its N-terminal long loop (e.g., in $E$. coli and Salmonella enterica); rhamnosylation of EF-P harboring an arginine at the same position (e.g., in Shewanella oneidensis and Pseudomonas aeruginosa); and 5-amino-pentanolylation of EF-P containing a lysine also at the same position in Bacillus subtilis (Rossi et al. 2014).

The crystal structure of unmodified Thermus thermophilus EF-P bound to the 70S ribosome and initiator formylmethionyl-tRNA $\mathrm{i}_{\mathrm{i}}$ has been determined, revealing a binding site between the $\mathrm{P}$ and $\mathrm{E}$ sites of the ribosome, contacting ribosomal protein L1, rRNA close to the peptidyl transferase center (PTC) and the peptidyl site tRNA (P-tRNA) (Blaha et al. 2009). More recently, the structure of S. cerevisiae modified eIF5A in complex with the ribosome and P-tRNA was determined by cryo-electron microscopy (Schmidt et al. 2015) and crystallography (Melnikov et al. 2016). In addition to this eukaryotic complex exhibiting similar aspects as the EF-P + ribosome + P-tRNA, the hypusine directly interacts with the CCA end of the P-tRNA. These data suggest that the essential role of hypusine in eIF5A is to stabilize the correct positioning of the CCA end of the P-tRNA to assist peptide bond formation by the PTC.

Nonetheless, no systematic analysis has been performed so far to determine the important residues on the surface of eIF5A that are required for the binding to ribosomal complexes. In this study, we used clustered charged-toalanine mutagenesis and modeling of the eIF5A + ribosome + P-tRNA complex to better understand the structural requirements of eIF5A for ribosome binding.

\section{Materials and methods}

\section{Generation of plasmids encoding eIF5A site-directed mutants from alanine scanning}

To generate the new eIF5A mutants described herein, the plasmid pYGEX-TIF51A (Cano et al. 2008; Koepp et al. 1996) was used as the template for mutagenesis by the QuickChange Site-direct Mutagenesis Kit (Stratagene), according to the manufacturer's instructions. The complete open reading frame was sequenced to validate the intended mutations.

\section{Molecular modeling and complex structural analysis}

The eIF5A structure was previously obtained by crystallography and deposited on Protein Data Bank PDB 3ER0. This structure presents a monomer chain at $3.35-\AA$ resolution, but missing some $\mathrm{N}$-terminal, $\mathrm{C}$-terminal and hypusination loop residues. Because of these missing residues, molecular modeling was performed to obtain a complete model of eIF5A. The sequence GI: 215,794,766 was submitted to I-TASSER (Yang et al. 2015) using itself as a template (PDB ID 3ER0:A).

Saccharomyces cerevisiae ribosome structure $\mathrm{PDB}$ 4V7R (Ben-Shem et al. 2010) was used for eIF5A-ribosome complex analysis performed by structural alignment using Pymol, Version 1.3 (Schrodinger, LLC) based on crystallographic and cryo-EM structures of the ribosome in complex with tRNAs at the peptidyl and exit sites (abbreviated as $\mathrm{P}$ and $\mathrm{E}$, respectively).

Initially, the cryo-EM structure from the Plasmodium falciparum $80 \mathrm{~S}$ ribosome bound to P/E-tRNA, PDB 3JBO (Sun et al. 2015), was used to coordinate references and align the $80 \mathrm{~S}$ in complex with P-tRNA (PDB 3JBN). Next, the structure of the $40 \mathrm{~S}$ ribosome from $T$. thermophilus bound to EF-P, PDB 4V6A (Blaha et al. 2009) was three dimensionally aligned using the tRNA at the P-site as the coordinates reference.

Then, then $80 \mathrm{~S}$ ribosome from yeast was aligned to the previously superposed structures to hide other structures and retain only EF-P and tRNA at the P-site (PDB 4V6A) for subsequent analyses.

Finally, the eIF5A model was aligned to the EF-P structure resulting in the 80S S. cerevisiae ribosome in complex with tRNA at the P-site and eIF5A. The analysis of cleavage sites was performed using a 25S RNA sequence (PDB 3058) based on regions previously described (Gutierrez et al. 2013), and the human eIF5A crystallographic structure, PDB 3CPF (Tong et al. 2009), was aligned to eIF5A to corroborate the structure analyses.

\section{Cell growth assay and complementation analyses in yeast}

The eIF5A mutants were generated in pYGEX-TIF51A under the $G A L 1$ promoter (galactose inducible). The strain VZL1074 (MATa ura3 leu2 his3 kanMX4-tetOpr-TIF51A ura3::CMV-tTA), with endogenous eIF5A expression controlled by a tetracycline repressible promoter, was transformed with plasmids containing the new eIF5A mutants (pSV1373, pSV1476, pSV1479 and pSV1482) and used 
for the growth analyses. Briefly, liquid cultures were grown in $5 \mathrm{~mL}$ of medium SC-ura containing raffinose to suppress the plasmid expression until the exponential phase $\left(\mathrm{DO}_{600 \mathrm{~nm}}=0.6-0.8\right)$. A sample of the culture was centrifuged at $600 \times g$ for $7 \mathrm{~min}$, and the pellet was suspended in $50 \%$ glycerol to a final concentration of $2.5 \times 10^{8}$ cells/ $\mathrm{mL}$. Tenfold serial dilutions were plated onto media SCura containing tetracycline and glucose (in the absence of eIF5A) or galactose (expressing heterologous eIF5A). The plates were incubated at $25^{\circ} \mathrm{C}$ for 3-4 days.

\section{Detection of hypusine-containing eIF5A}

To analyze the presence of hypusine-containing eIF5A, cultures of the yeast strain VZL1074 transformed with plasmids containing the new eIF5A mutants were grown overnight at $25{ }^{\circ} \mathrm{C}$ in $5 \mathrm{~mL}$ of SC-ura media with raffinose, and then the cells were transferred into SC-ura media containing tetracycline and galactose to express the eIF5A mutants. After $6 \mathrm{~h}$ of induction, samples were lysed in protein lysis buffer A (50 mM Tris-HCl pH 7.5; $50 \mathrm{mM}$ dithiothreitol; 5 mM EDTA; $5 \%$ SDS; $5 \%$ glycerol; $2 \times$ Protease Inhibitor Cocktail), and $15 \mu \mathrm{g}$ of whole cell extracts (WCE) were resolved by SDS-PAGE and transferred to a nitrocellulose membrane. We proceeded to perform immunoblot analysis using a rabbit polyclonal anti-eIF5A (yeast) antibody at a 1:15,000 dilution (Valentini et al. 2002), a rabbit polyclonal anti-hypusine antibody (Millipore) at a 1:1500 dilution (Mandal et al. 2015) and a rabbit polyclonal anti-RPL5 (yeast) antibody at a 1:15,000 dilution with an enhanced chemiluminescence detection system on a Li-Cor C-Digit Blot Scanner.

\section{Glutathione-S-transferase (GST) pulldown assay}

The yeast strains VZL1074 expressing GST (pSV20), GST-wild-type eIF5A (pSV36) or GST-eIF5A mutants (pVZ1476, pVZ1479, pVZ1482) were grown in SCura $+2 \%$ galactose until $\mathrm{DO}_{600 \mathrm{~nm}}=0.8$. Then, cells were disrupted in lysis buffer $\mathrm{B}(20 \mathrm{mM}$ Tris- $\mathrm{HCl} \mathrm{pH}$ 7.5; KOAc $50 \mathrm{mM} ; \mathrm{MgCl}_{2} 10 \mathrm{mM}$; DTT $1 \mathrm{mM}$ and $1 \mathrm{mM}$ Protease Inhibitor Cocktail) by agitation in Fast Prep equipment (three $25 \mathrm{~s}$ agitations) with glass beads. The cells were clarified by centrifugation at $20,000 \times g$ for $15 \mathrm{~min}$ at $4{ }^{\circ} \mathrm{C}$, and the lysates subjected to GST pulldown as previously published (Zanelli et al. 2006). Briefly, clarified lysates $(2 \mathrm{mg})$ were adjusted to the same volume and incubated with $100 \mu \mathrm{L}$ of glutathione-Sepharose beads (50\% slurry) for $1 \mathrm{~h}$, at $4{ }^{\circ} \mathrm{C}$. Beads were collected, washed five times with cold buffer B, and resuspended in $30 \mu \mathrm{L}$ of SDSPAGE loading buffer. Immunoblot analysis of WCE and bound fractions from GST-eIF5A and GST alone was performed using the rabbit polyclonal antibodies anti-eIF5A
$(1: 10,000)$ and anti-RPL5 $(1: 15,000)$. Both anti-eIF5A and anti-RPL5 were raised in rabbit against GST-fusion proteins purified from bacteria, so they also recognize GST.

\section{Expression and purification of eIF5A protein versions}

The yeast hypusine-containing eIF5A (eIF5A-Hyp) and non hypusine-containing eIF5A (eIF5A-Lys) were expressed and purified according to a modified protocol (Park et al. 2011). Briefly, 2 L of BL21(DE3)groES (Takara Bio) cells containing a polycistronic vector coexpressing the yeast genes of the hypusination modifying enzymes (DYSI and LIAI) and pET28a-eIF5A versions (which yields $6 \times$ His-eIF5A) were harvested after $16 \mathrm{~h}$ of induction at $18^{\circ} \mathrm{C}$. Cells were then lysed by sonication in ice cold Buffer C (50 mM Tris-Cl pH 7.0; $0.1 \mathrm{mM}$ EDTA; $300 \mathrm{mM} \mathrm{KCl} ; 1 \mathrm{mM}$ DTT; $20 \mathrm{mM}$ imidazole). The lysate was mixed with $5 \mathrm{~mL}$ of equilibrated NiNTA resin (Qiagen), and proteins were eluted in Buffer D (Buffer C containing $250 \mathrm{mM}$ imidazole). The eluate was dialyzed against Buffer E (50 mM Tris-Cl pH 7.0; 0.1 mM EDTA; $1 \mathrm{mM}$ DTT), and cationic chromatography in SP column was performed onto equilibrated $5 \mathrm{~mL}$ HiTrap-SP column (GE Healthcare) to separate eIF5A-Hyp and eIF5A-Lys (Park et al. 2011). Proteins were eluted from the HiTrapSP column in a gradient of $0-60 \%$ of Buffer F $(50 \mathrm{mM}$ Tris-Cl pH 7.0; 0.1 mM EDTA; $10 \%$ glycerol; $1 \mathrm{M} \mathrm{KCl;}$ $1 \mathrm{mM}$ DTT), and the fractions containing eIF5A-Lys (pI lower and first peak) and eIF5A-Hyp (pI higher and second peak) were collected and analyzed by SDS-PAGE. Purified proteins were then concentrated using Amicon Ultra $3 \mathrm{kDa}$ (Merck Millipore), immediately frozen in liquid nitrogen and maintained at $-80{ }^{\circ} \mathrm{C}$.

Yeast ribosomes were prepared based on previous protocols (Ben-Shem et al. 2010; Fraser et al. 2007) with some modifications. Approximately, $30-40 \mathrm{~g}$ of cells from early saturated culture of 1-2 OD were immediately frozen in liquid nitrogen and lysed in $40 \mathrm{~mL}$ of lysis Buffer $\mathrm{F}\left(20 \mathrm{mM}\right.$ Hepes pH 7.5; $100 \mathrm{mM} \mathrm{KCl}, 5 \mathrm{mM} \mathrm{Mg}(\mathrm{OAc})_{2}$, $1 \mathrm{mM}$ DTT, protease inhibitors). Cells were split into three to four round-bottom tubes containing $20 \mathrm{~g}$ of glass beads and vortexed for six cycles of $1 \mathrm{~min} \mathrm{ON}-1 \mathrm{~min}$ OFF at $4{ }^{\circ} \mathrm{C}$. Twenty-five milliliters of clarified lysate were loaded on the top of a $20 \%$ sucrose cushion $(20 \mathrm{mM}$ Hepes $\mathrm{pH}$ $7.5 ; 100 \mathrm{mM} \mathrm{KCl} ; 5 \mathrm{mM} \mathrm{Mg}(\mathrm{OAc})_{2} ; 1 \mathrm{mM}$ DTT) in a Beckman Ti45 tube. The purification of ribosomes and the separation of $40 \mathrm{~S}$ and $60 \mathrm{~S}$ subunits were performed as previously described (Fraser et al. 2007).

\section{Protein labeling with fluorescein}

The purified single-cysteine version of human eIF5A, eIF5A $\mathrm{A}^{\mathrm{C} 22 \mathrm{~A}, \mathrm{C} 38 \mathrm{~A}, \mathrm{C} 73 \mathrm{~A}, \mathrm{C} 129 \mathrm{~A}, \mathrm{T1} 142 \mathrm{C}}$ (eIF5A ${ }^{\mathrm{T} 142 \mathrm{C}}$ ), was labeled 
Table 1 Values of mean and standard deviation of the anisotropy change and equilibrium binding constants $K_{\mathrm{i}}$

\begin{tabular}{llllr}
\hline Protein-Fl $+80 \mathrm{~S}$ & $K_{\mathrm{i}}(\mathrm{nM})^{\mathrm{a}}$ & $r_{\text {free }}$ or $r_{\text {min }}{ }^{\mathrm{b}}$ & \multicolumn{1}{c}{$r_{\text {bound }}{ }^{\mathrm{c}}$} & \multicolumn{1}{c}{$\Delta r_{\max }{ }^{\mathrm{d}}$} \\
\hline TIF51A-Hyp & $9 \pm 1.1$ & $0.177 \pm 0.01$ & $0.245 \pm 0.005$ & $0.068 \pm 0.006$ \\
tif51A-4-Hyp & $171 \pm 14$ & $0.191 \pm 0.003$ & $0.261 \pm 0.010$ & $0.070 \pm 0.013$ \\
tif51A-6-Hyp & $167 \pm 54$ & $0.211 \pm 0.007$ & $0.252 \pm 0.011$ & $0.0413 \pm 0.008$ \\
tif51A-7-Hyp & $1148 \pm 508$ & $0.175 \pm 0.015$ & $0.255 \pm 0.004$ & $0.080 \pm 0.018$ \\
TIF51A-Lys & $238 \pm 43$ & $0.201 \pm 0.005$ & $0.244 \pm 0.001$ & $0.043 \pm 0.004$ \\
tif51A-4-Lys & $388 \pm 71$ & $0.201 \pm 0.020$ & $0.256 \pm 0.006$ & $0.054 \pm 0.004$ \\
tif51A-6-Lys & nd & $0.206 \pm 0.026$ & $0.243 \pm 0.009$ & $0.037 \pm 0.035$ \\
tif51A-7-Lys & nd & $0.229 \pm 0.004$ & $0.236 \pm 0.004$ & $0.007 \pm 0.001$ \\
\hline
\end{tabular}

nd not determined measurements

a Equilibrium binding constant obtained by titrating eIF5A as a competitor into fixed amount of ribosome + eIF5A-fluorescein

b Anisotropy values for the free eIF5A-fluorescein or the minimum anisotropy detected for the competition assays

${ }^{c}$ Anisotropy of the eIF5A-fluorescein bound to the ribosomal complex in the absence of competitors

d Maximum anisotropy change obtained by the difference between $r_{\text {free }}$ or $r_{\min }$ and $r_{\text {bound }}$ with fluorescein-5-maleimide (Thermo Scientific), following published protocols (Sokabe and Fraser 2014). After labeling, eIF5A-fluorescein was purified from the free dye by spinning in a $2 \mathrm{~mL}$ size exclusion column of $6 \mathrm{kDa}$ (BioRad). The amounts of dye and protein were evaluated by $495 \mathrm{nM}$ absorbance and quantitative dot blots using wild-type eIF5A as a standard. Only the preparation with more than $95 \%$ of labeling was used for fluorescence anisotropy assays.

\section{Fluorescence anisotropy}

Competition assays with fluorescein-labeled eIF5A (eIF5A-fluorescein) were conducted using a VICTOR X5 Multilabel Plate Reader (Perkin Elmer) and a previously published protocol (Rossi et al. 2016). Inhibition constants $\left(K_{\mathrm{i}}\right)$ of non-labeled wild-type and mutant eIF5A versions were determined using a in vivo pre-assembled $80 \mathrm{~S}$ ribosome (heterogeneous mixture of yeast 80S) in complex with eIF5A-fluorescein, in which the concentration of the ribosome and eIF5A-fluorescein was held constant at 30 and $5 \mathrm{nM}$, respectively, and the starting concentration of the eIF5A competitors was $5 \mu \mathrm{M}$. The addition of higher concentrations of the competitor did not change the equilibrium inhibition constant values, indicating that the reaction was prepared in saturating conditions. Anisotropy binding reactions were set up in $22 \mu \mathrm{L}$ final volume in reaction buffer $[20 \mathrm{mM}$ Hepes $\mathrm{pH} 7.5 ; 100 \mathrm{mM}$ KOAc; $2.5 \mathrm{mM} \mathrm{Mg}(\mathrm{OAc})_{2} ; 1 \mathrm{mM}$ DTT; $\left.15 \mu \mathrm{M} \mathrm{BSA}\right]$, and increasing concentrations of competitors were added, as indicated in the legend of Fig. 6. Twenty microliters of each reaction was transferred to a 384 -well plate and incubated at $30{ }^{\circ} \mathrm{C}$ for $30 \mathrm{~min}$ to reach equilibrium. Fluorescence-polarized (FP) light and total fluorescence were measured, and the anisotropy change $(r)$ was converted into the fraction of eIF5A-fluorescein bound to the ribosome and fitted to the solution of a quadratic equation describing an equilibrium reaction (Sokabe and Fraser 2014). The values presented in Table 1 were obtained from the average of three independent replicates, and the errors provided are standard deviations (SD) of the data. All data are expressed as mean values $\pm \mathrm{SD}$ and analyzed by two-tailed Student's unpaired $t$ test. In all tests, differences were considered significant at $p<0.05$.

\section{Results and discussion}

\section{eIF5A contains four clusters of charged residues potentially important for interaction with ribosomal components}

To search for residues on the surface of eIF5A that are important for binding to the ribosome, we combined the clustered charged-to-alanine scanning mutagenesis strategy and molecular modeling of the eIF5A-ribosome complex. The cluster charged to alanine scanning mutagenesis is based on the replacement of clustered charged amino acid residues (E, D, K, R and $\mathrm{H}$ ), which are possible sites of protein-protein or protein-RNA interactions, by alanine across all protein sequences.

In this work, we searched for clusters of five amino acid residues present in the eIF5A primary sequence containing at least two charged residues and substituted the charged residues with alanine (Fig. 1a). We excluded from our analysis only the charged clusters inside the hypusine loop of eIF5A (from residues 45 to 60), because they are already known to be necessary for hypusine formation, which in 


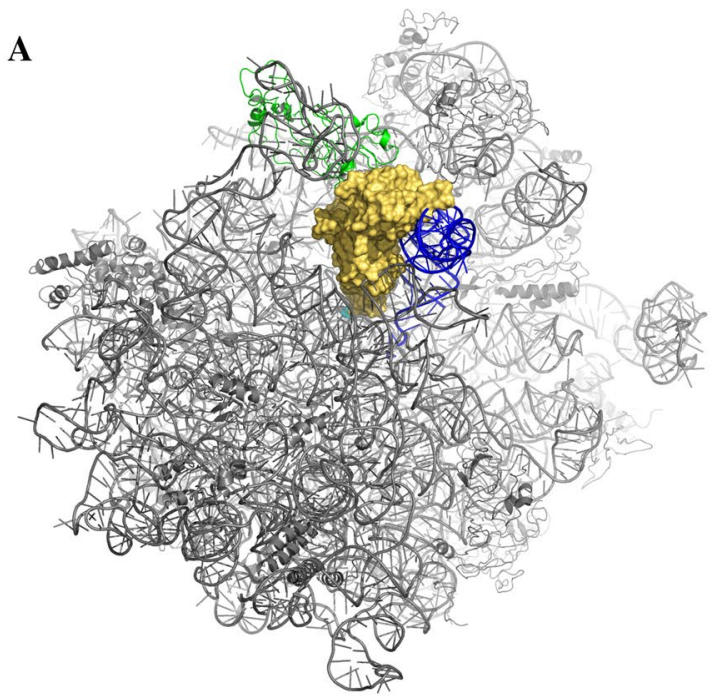

C

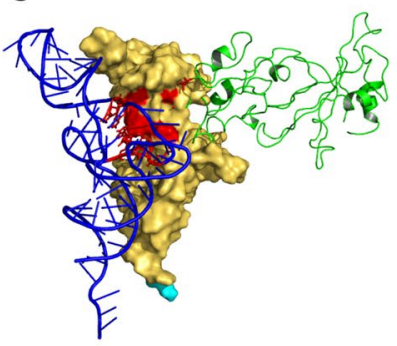

cluster 4

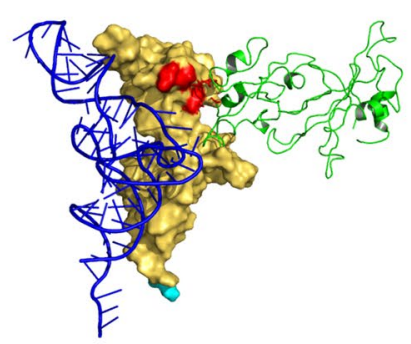

cluster 6

Fig. 2 Model of eIF5A binding to 60S-tRNA ribosome complex. a Interaction model of surface representation of $S$. cerevisiae eIF5A (yellow) with hypusination site in cyan (PDB 3ER0) and ribbon representation of Met-tRNA ${ }_{i}^{\text {Met }}$ (blue; PDB 3JBN) on the ribbon structure of yeast $60 \mathrm{~S}$ ribosome (gray; PDB $4 \mathrm{~V} 7 \mathrm{R}$ ). The position of $\mathrm{L} 1$ is

turn is necessary for ribosome binding (Cano et al. 2008; Dias et al. 2008). We also excluded the $\mathrm{N}$ terminus (from residues 1 to 16 ) and $\mathrm{C}$ terminus (from residues 151 to 157), because they are not present in the crystal structure of eIF5A (PDB 3ER0). In this way, we designed 11 new eIF5A mutants, localized in different regions of the surface of eIF5A (Fig. 1b).

The eIF5A location on $80 \mathrm{~S}$ was modeled similarly as reported previously using data from tRNA and rRNA hydroxyl radical cleavage (Gutierrez et al. 2013). We performed the molecular modeling and complex structural analysis to investigate the distances of eIF5A residues from the ribosome and tRNA. Figure 2a shows the binding region of eIF5A (yellow) in the large subunit of the ribosome (gray), located between the $\mathrm{P}$ and $\mathrm{E}$ sites and the modification loop (cyan) near the peptidyl transferase center of the ribosome. Figure $2 b$ is a magnified view of the crystal structure of eIF5A bound to ribosomal 60S subunit protein L1 (L1) (green) and the P-site tRNA (blue). The location of eIF5A on 80S corroborates

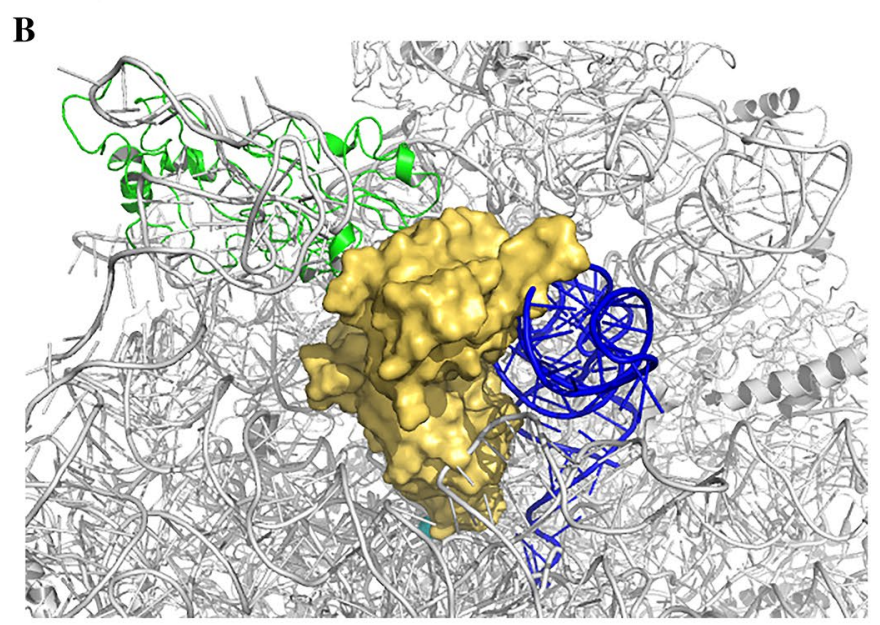

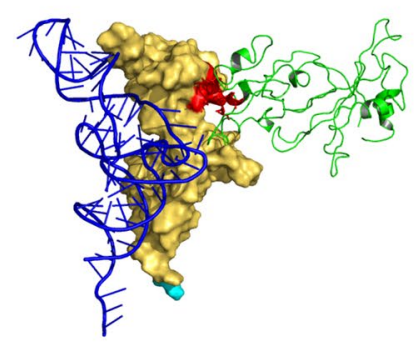

cluster 7

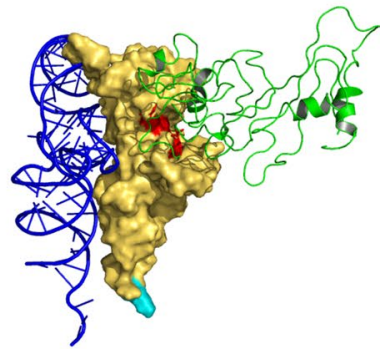

cluster 11 indicated in green. b Magnified views of docked eIF5A, P-site MettRNA $_{i}^{\text {Met }}$ and L1 protein. c Magnified views of docked eIF5A, P-site Met-tRNA $\mathrm{i}_{\mathrm{i}}^{\mathrm{Met}}$ and L1 protein, with the amino acid residues mutated highlighted in red, corresponding to the indicated cluster

with the location of its structural homolog in bacteria, EF-P on the 70S ribosome (Blaha et al. 2009), and our model is consistent with the previously obtained model (Gutierrez et al. 2013). We then used this model to analyze the 11 charged clusters of the eIF5A surface, and clusters 4, 6, 7 and 11 were selected for further analysis because they not only harbor residues predicted to physically contact the rRNA to some extent, but also show more evidence for binding to the L1 protein (clusters 6 , 7 and 11) or the P-site tRNA (cluster 4) (Fig. 2c, data not shown).

Since two independent structures of eIF5A in complex with the ribosome were published (Schmidt et al. 2015; Melnikov et al. 2016) while we prepared this work, we also considered their results. Although both structures are in general agreement with our model (Supplementary figures S1), only the coordinates for the cryo-electron microscopy (Schmidt et al. 2015) have been released so far. Comparing more specifically the clusters $4,6,7$ and 11 , only cluster 11 does not demonstrate residues interacting with the 


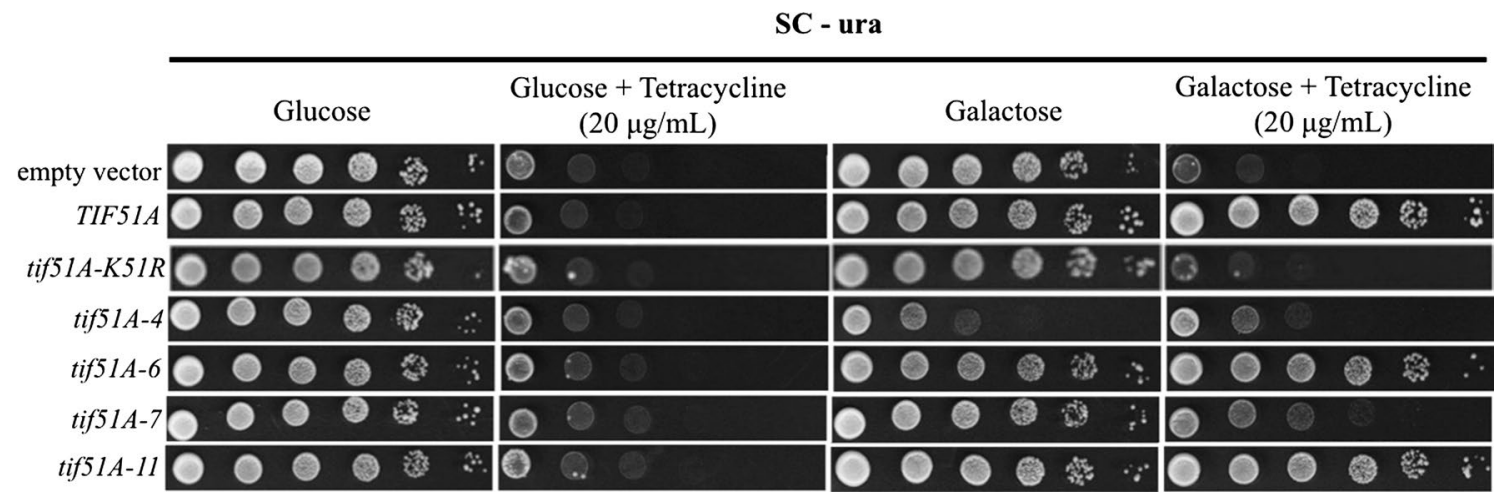

Fig. 3 Growth analysis of $S$. cerevisiae expressing eIF5A mutants from alanine scanning. Serial dilutions of VZL1074 strain (tetOprTIF51A) containing pSV20 (vector pYGEX - negative control), pSV36 (pYGEX-TIF51A-positive control), pSV41 (pYGEX-

ribosome, as predicted in our model. That is, in the cryoEM structure, cluster 4 also shows interaction with the P-site tRNA and clusters 4, 6 and 7 also physically contact the L1 protein (Supplementary Figure S2).

\section{Clustered charged-to-alanine mutants tif51A-4 and tif51A-7 of eIF5A exhibit important growth defects not related to decreased eIF5A protein or hypusine levels}

To assess the functional effects of changing the charged residues present in clusters $4,6,7$ and 11 , we tested their abilities to complement a yeast strain depleted of eIF5A. The mutations to substitute the charged residues for alanine were constructed in the plasmid pYGEX-TIF51A (Cano et al. 2008; Koepp et al. 1996), which is under the control of galactose for expression, and introduced in the strain VZL1074 (tetOpr-TIF51A), which has eIF5A production turned off in the presence of tetracycline. These new alleles of eIF5A, containing charge clustered-to-alanine mutations, were named after their cluster names: tif51A-4, tif51A-6, tif51A-7 and tif51A-11. Figure 3 illustrates the results of complementation of these eIF5A mutants, with negative (empty vector pYGEX), positive (pYGEX-TIF51A) and the non-hypusinatable mutant eIF5 ${ }^{\mathrm{K} 51 \mathrm{R}}$ (pYGEXtif51 ${ }^{\mathrm{K} 51 \mathrm{R}}$ ) as controls (Schnier et al. 1991). As observed in Fig. 3, the mutants tif51A-6 and tif51A-11 could complement the absence of wild-type eIF5A, whereas the mutants tif51A-4 and tif51A-7 exhibited an important reduction in their abilities to promote growth in these conditions. Although the mutants tif51A-4 and tif51A-7 could maintain some growth in the absence of wild-type eIF5A compared with the empty vector and $t i f 51 A^{\mathrm{K} 51 \mathrm{R}}$ mutant, their growth phenotypes clearly demonstrated that the substitutions of the clustered charged residues to alanine in these mutants impaired eIF5A function. In addition to complementation, tif51 $1^{\mathrm{K} 51 \mathrm{R}}$ —non-hypusinatable control) or mutants tif51A-4, tif51A-6, tif51A-7 and tif51A-11 were inoculated in SC-ura supplemented with or without $20 \mu \mathrm{g} / \mathrm{mL}$ tetracycline, containing $2 \%$ glucose or galactose, and incubated at $25^{\circ} \mathrm{C}$ for 3 days

this assay could also evaluate the negative dominance of these mutants when there was simultaneous expression of wild-type and mutant eIF5A (tetracycline-free medium containing galactose). Curiously, the mutant tif51A-4 showed a dominant negative phenotype, since a growth defect was observed even in the presence of the wild-type gene (SC-ura + galactose). The finding of a dominant negative mutant of eIF5A is quite interesting, because there is no eIF5A dominant negative mutant described so far. This dominant negative can be useful in future genetic screenings in the presence of endogenous eIF5A.

Because the impairment of growth in mutants tif51A-4 and tif51A-7 may be a result of a decrease in total eIF5A protein levels, we tested mutant eIF5A levels compared to the wild-type eIF5A levels. As observed in the left panel of Fig. 4 (total eIF5A) and the WCE lanes of Fig. 5a, both mutants tif51A-4 and tif51A-7 produced levels of modified eIF5A protein similar to the wild type, indicating that their inability to complement is caused by the lack of eIF5A function, rather than by the loss of the mutant protein.

Additionally, because hypusine modification is essential for eIF5A activity (Park 2006), we examined whether the growth defects of the eIF5A mutants could be related to decreased hypusination levels. To determine if the eIF5A mutants were hypusinated in vivo, we performed an immunoblot assay using an antibody that specifically recognizes hypusinated eIF5A and compared these results with total eIF5A. Wild-type eIF5A and the non-hypusinatable eIF5 ${ }^{\mathrm{K} 51 \mathrm{R}}$ mutant (Schnier et al. 1991) were used as controls. The hypusination levels of eIF5A and the mutants were similar in this assay, and this information further supports the idea that the defective growth phenotype of the tif51A-4 and tif51A-7 mutants is not related to their hypusination levels (Fig. 4).

We also compared the complementation results obtained for the new eIF5A mutants to a previously published systematic mutational analysis of human eIF5A, which also 


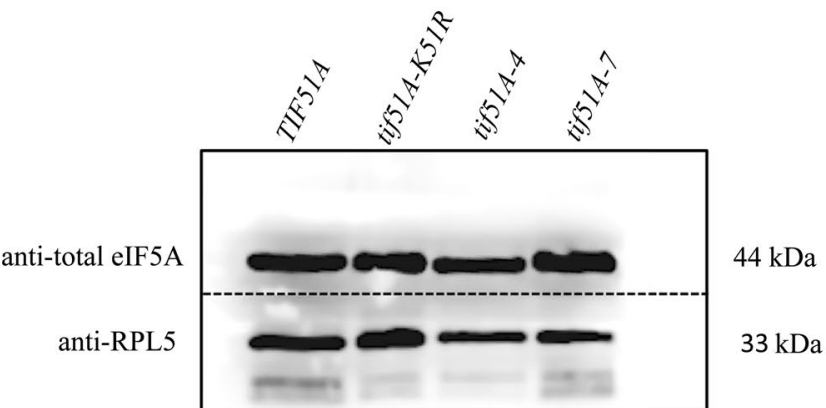

Fig. 4 Charged clustered-to-alanine tif51A-4 and tif51A-7 mutants exhibited no decrease in total eIF5A or hypusination levels. The levels of total eIF5A and hypusine-containing eIF5A were tested for the eIF5A produced by mutants tif51A-4 and tif51A-7 using an eIF5A antibody that recognizes all eIF5A forms or the hypusine-specific

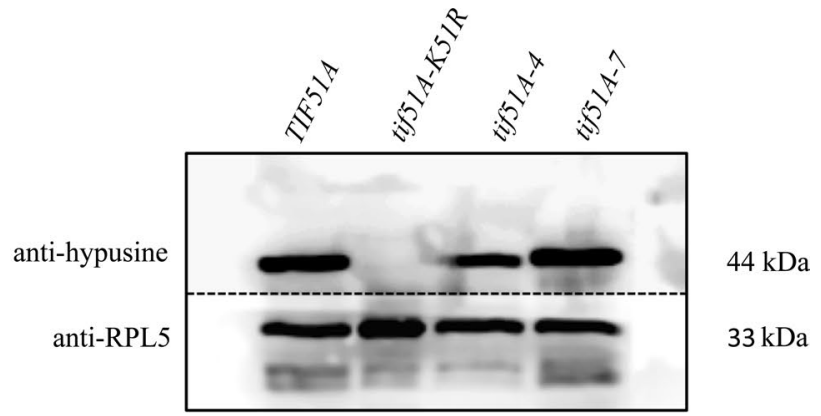

eIF5A antibody. An anti-RPL5 was used as loading control. The allele producing the eIF5A analyzed is indicated on the top of each lane. The tif51A-K51R allele generates a non-hypusinatable eIF5A (Schnier et al. 1991)

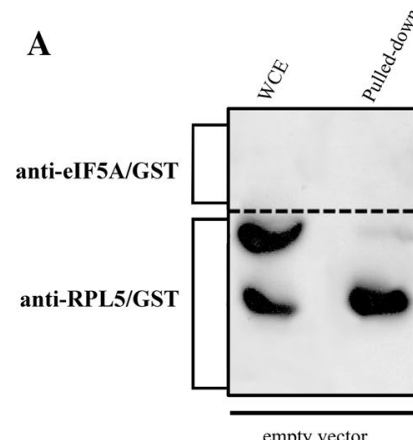

B
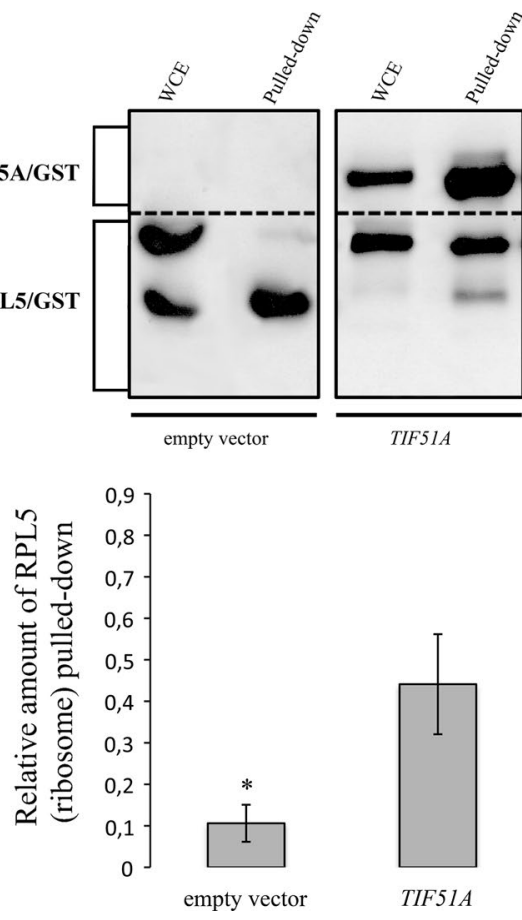

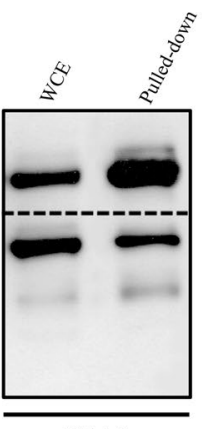

tif51A-4

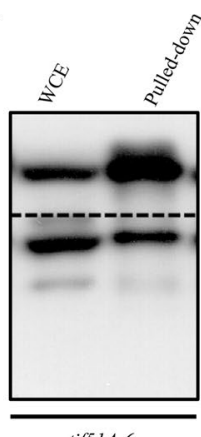

tif5IA-6

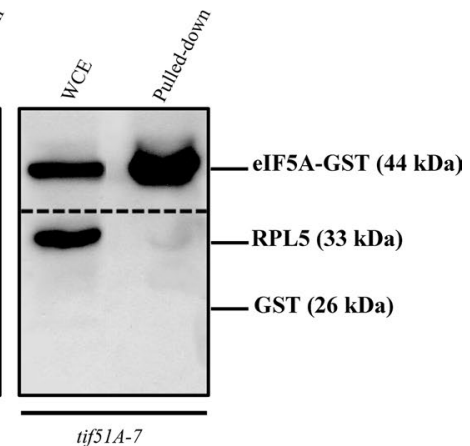

Fig. 5 GST pulldown of clustered charged-to-alanine mutants of eIF5A. a Yeast whole cell extracts (WCE) derived from cells expressing either GST alone or GST-eIF5A (from wild-type TIF51A, or from mutants tif51A-4, tif51A-6 and tif51A-7) were incubated with glutathione-Sepharose beads, washed, and proteins bound to the beads (bound) were denatured and fractionated by SDS-PAGE. Equal amounts of WCE and bound samples were loaded in the indicated lanes. Western blot analysis from fractions of whole cell extract (WCE) and bound ones was performed using specific polyclonal anti-

used substitution of residues to alanine. However, that study analyzed highly conserved residues (Cano et al. 2008), with a different criterion than ours. Considering the clusters of five amino acid residues shown in Fig. 1a, the study of human eIF5A analyzed the mutants P37A, R86A, P115A, bodies against ribosomal protein L5 (anti-L5) and anti-eIF5A. Both anti-eIF5A and anti-RPL5 were raised in rabbit against GST-fusion proteins purified from bacteria, so they also recognize GST. b GSTpulldown analysis of the same cells was carried out and the quantification of bands were performed to show the levels of ribosome pulled down. The error bars represent the standard deviation of the experimental values for three independent replicates and the data are expressed as mean values \pm SD. In all tests, differences were considered significant at $p<0.05$ using the wild type as control

E116A, L119A and E144A, overlapping, respectively, with the regions covered by clusters $2,4,8,8,9$ and 11 . The results for most of the single mutants and the clusters are the same, showing no phenotype (Fig. 3, data not shown) (Cano et al. 2008). Interestingly, for the mutant R86A, 

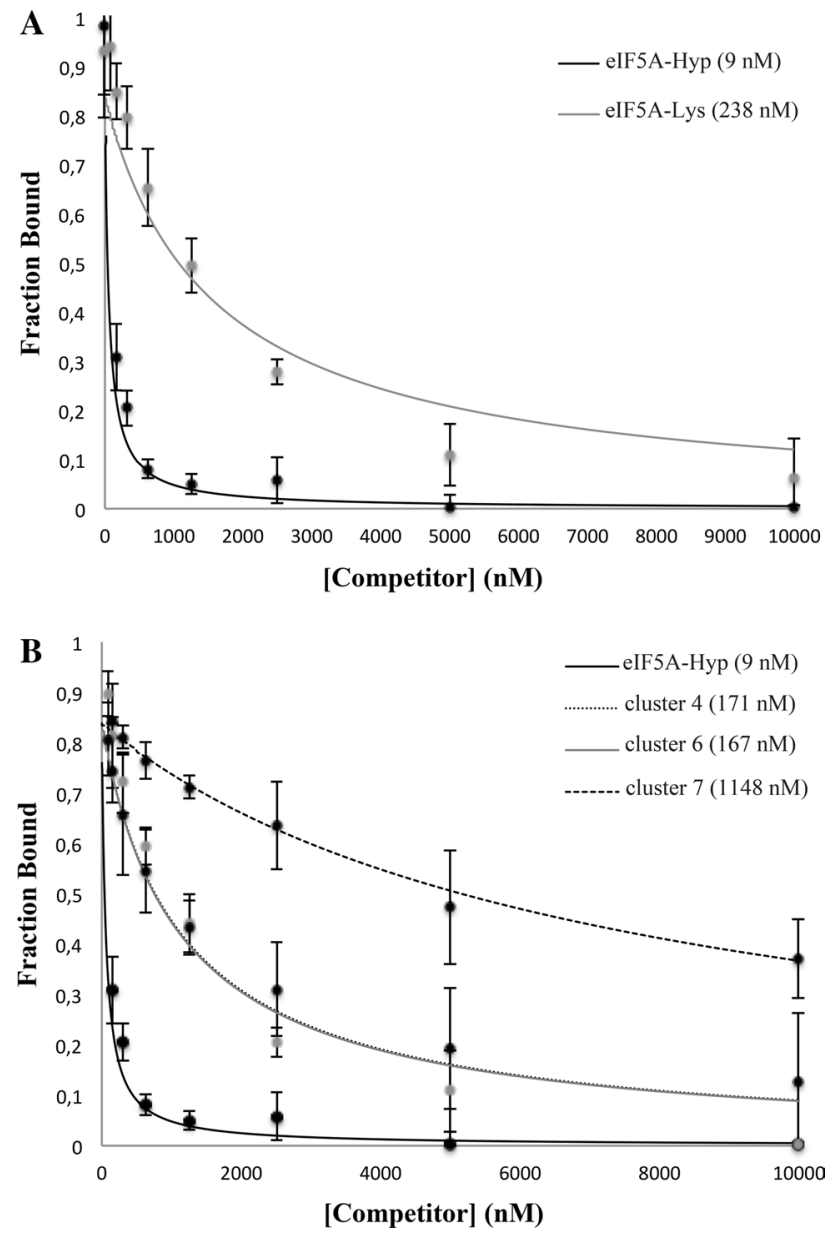

Fig. 6 Binding assays of eIF5A mutants with yeast 80 S ribosome. The curves in the graphs indicate the fraction of wild-type eIF5Afluorescein bound to the yeast $80 \mathrm{~S}$ in competition with: a wild-type eIF5A both containing hypusine (eIF5A-Hyp) or not (unmodifiedeIF5A-Lys) or b hypusine-containing eIF5A from wild type (TIF51A) or mutants tif51A-4, tif51A-6 and tif51A-7. All data were obtained in independent experimental triplicate are expressed as mean values \pm SD and analyzed by two-tailed Student's unpaired $t$ test. In all tests, differences were considered significant at $p<0.05$. The mean of $K_{\mathrm{i}}$ values is shown in parenthesis. The values of equilibrium binding constants and anisotropy changes are provided in Table 1

which corresponds exactly to the substitution R87A present in tif51A-4 (K86A/R87A/E89A), no growth phenotype was observed, while tif51A-4 is dominant negative, which suggests that the R87A is not sufficient or necessary to generate the phenotype observed for tif51A-4.

\section{eIF5A from mutant tif51A-7 exhibits an importantly reduced affinity for the ribosome in vivo and in vitro}

To evaluate the in vivo physical interactions between the eIF5A mutants and ribosomal complexes, we first performed in vivo GST-pulldowns (Fig. 5). For this analysis, we chose the eIF5A mutants tif51A-4 and tif51A-7 because of their growth phenotypes, along with one of the mutants that exhibited no growth phenotype, tif51A-6, as a control. Notably, the mutants tif51A-4 and tif51A-6 could co-purify the ribosome (detected by L5) in a manner similar to the wild type, whereas the mutant tif51A-7 exhibited a significant reduction of ribosome pulled down. These results suggest that the decrease in ribosome binding by mutated eIF5A may be the cause of the tif51A-7 growth phenotype and, therefore, that the charged residues of cluster 7 are necessary for this physical interaction.

Although the pulldown results for the tif51A-6 allele were consistent with its ability to substitute for wild-type eIF5A, the mutant tif51A-4 exhibited the most severe growth phenotypes among the mutants studied herein, yet it co-purified with the ribosome. As already shown in Fig. 1b, according to the modeled eIF5A-ribosome complex, the charged residues in cluster 4 (modified to alanine in tif51A-4) contact the P-site tRNA. Accordingly, our results suggest that interfering with residues on the surface of eIF5A binding to the P-site tRNA does not abolish its binding to the ribosome. Moreover, the ability of eIF5A from tif51A-4 to bind to the ribosome while potentially blocking physical interaction with P-site tRNA could explain its dominant negative growth phenotype.

Then, we performed fluorescence anisotropy, a more quantitative, in vitro assay, to compare the affinities between eIF5A isoforms and the ribosome. Fluorescence anisotropy is based on the fact that when a protein conjugated to a fluorophore is excited in solution with polarized light, it emits fluorescence as polarized light dependent on its mobility (Lakowicz 2006). Therefore, free proteins have much higher mobility compared with proteins in a larger complex. The affinities of eIF5A-Hyp $\left(K_{\mathrm{i}} \approx 8 \mathrm{nM}\right)$ to the $80 \mathrm{~S}$ revealed that these proteins could bind directly and tightly to the 80S. As expected (Jao and Chen 2006; Zanelli et al. 2006), hypusine was required for a stable eIF5A-ribosome binding, because the Ki for eIF5A-Lys $\left(K_{\mathrm{i}} \approx 238 \mathrm{nM}\right)$ was approximately 30 times lower than eIF5A-Hyp. Table 1 provides the experimental values and standard deviations (SD) obtained from triplicate for minimum anisotropy $\left(r_{\text {min }}\right)$, maximum anisotropy $\left(r_{\text {bound }}\right)$, maximum anisotropy variation $\left(\Delta r_{\max }\right)$ and binding constant $\left(K_{\mathrm{i}}\right)$. Likewise, all non-modified (non-hypusine) mutant proteins (Lys) exhibited $K_{\mathrm{i}}$ values considerably lower than hypusine-containing proteins (Hyp) (Table 1). The affinities of eIF5A-Lys from mutants tif51A-6 and tif5 1A-7 to the ribosome were too low to calculate their $K_{\mathrm{i}}$ values. Finally, hypusine-containing eIF5A from mutants tif51A-4, tif51A-6 and tif51A-7 were evaluated in the same fluorescence anisotropy assay (Fig. 6b). All alanine scanning mutants tested showed a significant decrease in affinity to the ribosome, indicating that all charged clusters tested herein somehow affected the interaction with 
Fig. 7 Multiple alignment of eIF5A among different organisms. Multiple alignments of the amino acid sequences of eIF5A from the eukaryotes Saccharomyces cerevisiae (Sce), Schizosaccharomyces pombe (Spo), Drosophila melanogaster (Dme), Mus musculus (Mmu), Homo sapiens (Hsa-isoforms I and II), Leishmania mexicana (Lme), Leishmania braziliensis (Lbr) and Caenorhabditis elegans (Cel), respectively. The asterisk indicates the lysine residue converted to hypusine. The horizontal arrows for $\beta$-strands and coils for helices shown above the alignments indicate the secondary structure from $S$. cerevisiae eIF5A structure (PDB ID 3ER0). The degree of conservation among the residues is represented by different colors, as indicated by the number inside the colored boxes. The vertical arrows mark the charged residues substituted by alanine

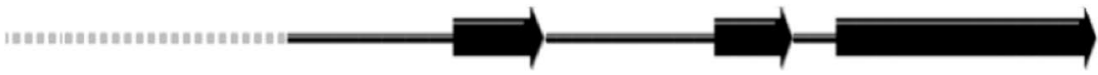

Sce

Spo

Dme

$\mathrm{Mmu}$

HsaI

Hsa I I

Lme

Lbr

Cel
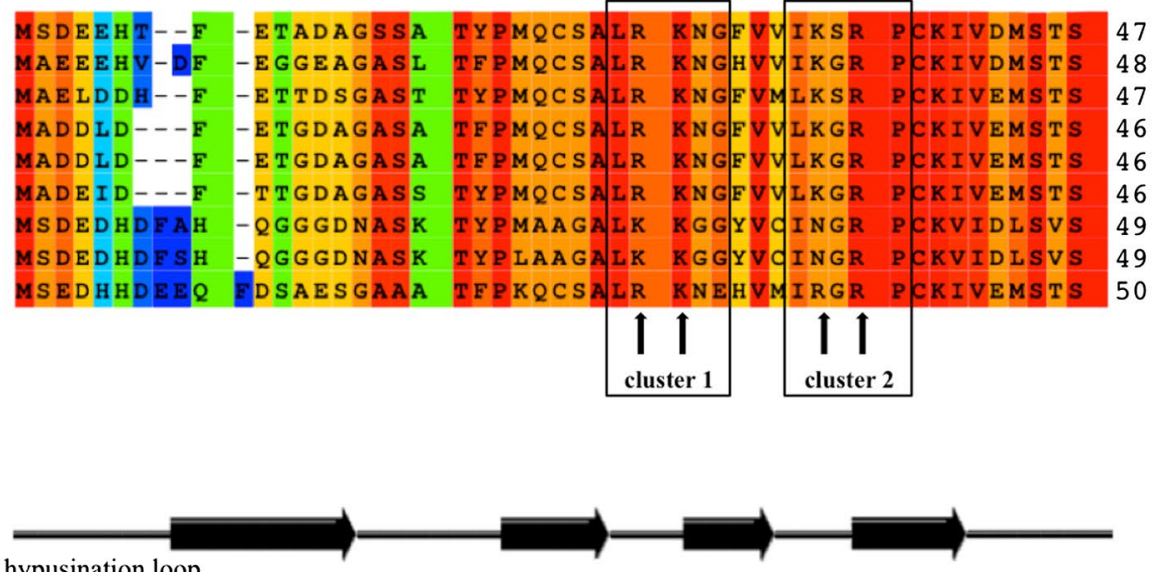

hypusination loop

\begin{tabular}{|c|c|c|c|c|c|c|c|c|}
\hline$\star$ & & & & & & & & \\
\hline KT GKHGHAKV & H LVA I D IF T G & K K LED & L S P S T & HNMEVPVV & $\mathrm{KR}$ & NEY & $2 \mathrm{~L} L$ & $\mathrm{DID}-$ \\
\hline KT TKHGHAKV & H I VAIDIFNG & RKY ED & MS P S T & HNMDVPVV & $\mathrm{KR}$ & DEY & $2 \mathrm{Lv}$ & N I D \\
\hline KTGKHGHAKV & HMVGIDIF S & KKY ED & I CP S T & HNMDVPNV & $\mathrm{K} R$ & EDL & $2 \mathrm{~L} I$ & $A$ \\
\hline KT TKHGHAKV & H L VGIDIFTG & K K Y E D & I CP S T & H NMDVPNI & KR & NDF & $2 \mathrm{I}$ & GIQ- \\
\hline KT TKHGHAKV & H LVGIDIFTG & K K Y ED & I CP S T & HNMDVPNI & $\mathrm{KR}$ & NDF & $2 \mathrm{I}$ & GIQ- \\
\hline KTGKHGHAKV & H L VG I D I F T & K K Y ED & I CP S T & H NMDVPNI & $\mathrm{KR}$ & NDY & $\mathrm{ex}$ & c \\
\hline KT GKHGHAKV & SIVATDIFTG & NRLED & 2 A P T & HNVEVPFV & $\mathrm{KT}$ & FTY & 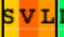 & DI \\
\hline KTGKHGHAKV & SIVATDIFTG & NRLED & QAP S T & HNVEVPFV & $\mathrm{KT}$ & Y T Y & S v L & DI \\
\hline KTGKHGHAKV & HMVAIDIFTT & KKLED & I CP S T & HNMDVPV & & REY & 4 & \\
\hline
\end{tabular}
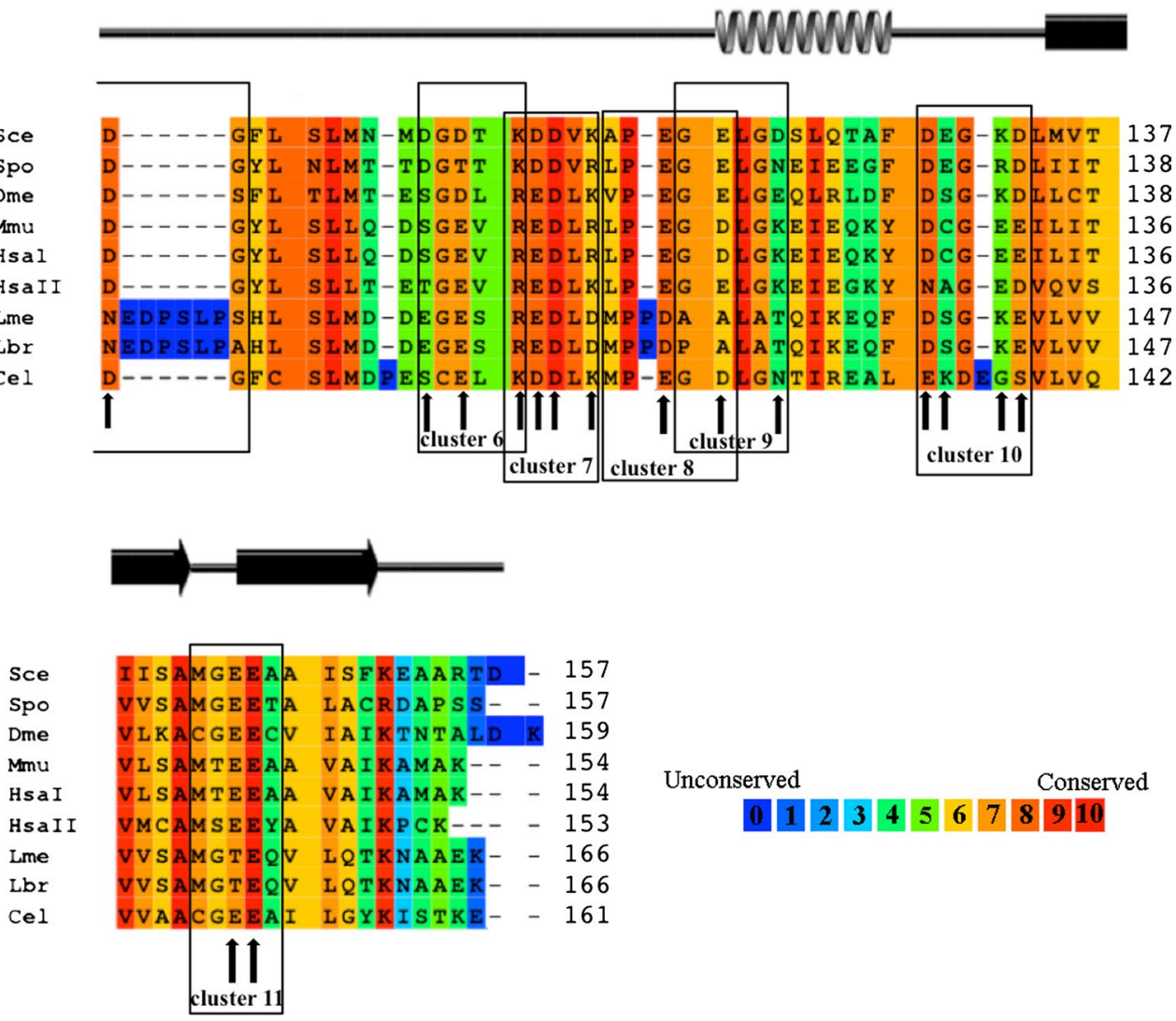

Unconserved Conserved

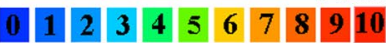


the ribosome, although not all of them exhibited growth phenotypes or revealed this decrease in affinity in the GST pulldown. Comparing the mutants among themselves, tif51A-4 $\left(K_{\mathrm{i}}=170 \mathrm{nM}\right)$ and tif51A-6 $\left(K_{\mathrm{i}}=167 \mathrm{nM}\right)$ exhibited similar binding affinities to the ribosome, whereas tif51A-7 exhibited the weakest ribosome binding $\left(K_{\mathrm{i}}>1 \mu \mathrm{M}\right)$, revealing a dramatic defect in ribosome binding by eIF5A from this mutant.

Notably, although the decrease in binding capacity of eIF5A from mutants tif51A-4 and tif51A-6 was not apparent in the GST-pulldown assay, it is possible that this less quantitative assay cannot clearly distinguish them from wild type. Additionally, the mutant tif51A-6 exhibiting no discernible growth phenotype suggests that a $\sim 10$-fold decrease in affinity binding of the eIF5A-ribosome complex does not compromise cell growth. This may really be the case, since eIF5A is the second most abundant translation factor in S. cerevisiae, occurring at an average concentration in the cell of about $10-15,000 \mathrm{nM}$, roughly the double of the ribosome concentration (Firczuk et al. 2013; Jorgensen et al. 2002; Kulak et al. 2014). Therefore, the in vivo eIF5A-ribosome binding conditions are highly saturating and the results from GST pulldown would better correlate with the results obtained for the highest eIF5A concentration points of Fig. $6 \mathrm{~b}$ than with the resulting $K_{\mathrm{i}}$ values themselves. From this analysis, we can conclude that mutants tif51A-4 and tif51A-6 are not different from the wild type at the highest concentrations of eIF5A $(10,000$ and 5,000 $\mathrm{nM})$; and mutant tif51A-4 is already not different from the wild type at $2500 \mathrm{nM}$ considering $95 \%$ or $99 \%$ confidence intervals (Supplementary Table). Thus, the results from GST pulldown and fluorescence anisotropy are not really conflicting and tend to correlate directly at higher concentrations of eIF5A.

Additionally, it is possible that other factors in vivo can contribute to alleviate this decrease in affinity of eIF5A to the ribosome in mutants tif51A-4 and tif51A-6. For example, our recent study demonstrated that eEF2 binding to the ribosome affects the binding of eIF5A in a negative manner (Rossi et al. 2016). Other factors in the cell might have a positive effect and mask the decrease observed in the fluorescence anisotropy.

Another important consideration is that the complementation and the GST-pulldown assays were performed using a galactose-inducible promoter, which may induce higher levels of eIF5A than those endogenously found in the cell. This over-expression could lead to the dominant negative phenotype of mutant tif $51 \mathrm{~A}-4$ or suppress the reduced ribosome-binding capacity of tif51A-6. Nevertheless, the observation that eIF5A from mutants tif51A-4 and tif51A-6 showed similar results in the anisotropy fluorescence assay further supports the idea that it is not decreased binding to the ribosome that generates the growth phenotypes in mutant tif51A-4. Finally, even considering a possible bias due to over-expression, this is still not enough to suppress the growth and ribosome binding defects of mutant tif51A-7, giving further support to the idea that it carries substitutions in residues central for eIF5A-ribosome physical interaction.

\section{Evolutionary conservation of eIF5A charged residues does not predict mutant phenotype}

We also investigated how conserved were the clusters mutated in this study and whether amino acid residue conservation and resultant mutant phenotype were directly correlated. As shown in Fig. 7, the multiple alignment of representative eukaryotic eIF5A sequences demonstrate the high overall evolutionary conservation of this protein, with the two sequences from the Leishmania species being the most divergent ones. We decided to include these two Leishmania eIF5A due to the fact that they had crystal structures solved. Considering the mutants tif51A-4 and tif51A-7 as the ones with the strongest phenotypes and mutants tif51A-1 and tif51A-11 the ones displaying no growth phenotypes, there is not a direct correlation between the conservation of the modified residues and mutant phenotype. For example, the degree of conservation of the charged residues in clusters 4 and 7 are lower than in cluster 3; however, the mutant derived from cluster 3 (tif51A-14) complements $S$. cerevisiae, while tif51A-4 and tif51A-7 do not. These results are in agreement with the previously published mutational analysis of human eIF5A (Cano et al. 2008) and demonstrate the need for the inclusion of more structural aspects of eIF5A for future mutational analyses.

Therefore, the structural model of eIF5A $+80 \mathrm{~S}+\mathrm{P}$-site tRNA we generated in this work, together with the analysis of possible interactions of clustered charged residues, lead us to uncover residues on eIF5A surface important for ribosome binding. One of these mutants, the tif51A-7, is unable to support growth and shows an importantly decreased binding capacity to the ribosome. The residues substituted in tif51A-7 are predicted to contact ribosomal protein L1, which has not been previously involved with eIF5A binding to the ribosome.

Acknowledgments We are grateful to Dr. Otavio H. Thiemann (Physics Institute of Sao Carlos, University of Sao Paulo-USP) for the use of computational laboratory infrastructure. This work was supported by grants to S. R. V. and C. F. Z. from the Fundação de Amparo à Pesquisa do Estado de São Paulo (FAPESP), the Conselho Nacional de Desenvolvimento Científico e Tecnológico (CNPq) and PADC from the Faculdade de Ciências Farmacêuticas, UNESP and CSF was supported by grant number R01GM092927 from National Institute of General Medical Sciences. We also thank the FAPESP for fellowships awarded to most of the authors. 


\section{Compliance with ethical standards}

Conflict of interest We declare that we have no conflicts of interest. This article does not contain any studies with human participants or animals performed by any of the authors.

\section{References}

Ben-Shem A, Jenner L, Yusupova G, Yusupov M (2010) Crystal structure of the eukaryotic ribosome. Science 330:1203-1209. doi:10.1126/science.1194294

Benne R, Hershey JWB (1978) Mechanism of action of protein-synthesis initiation-factors from rabbit reticulocytes. J Biol Chem 253:3078-3087

Blaha G, Stanley RE, Steitz TA (2009) Formation of the first peptide bond: the structure of EF-P bound to the 70S ribosome. Science 325:966-970

Cano VS et al (2008) Mutational analyses of human eIF5A-1identification of amino acid residues critical for eIF5A activity and hypusine modification. FEBS J 275:44-58. doi:10.1111/j.1742-4658.2007.06172.x

Dever TE, Green R (2012) The elongation, termination, and recycling phases of translation in eukaryotes. Cold Spring Harb Perspect Biol. doi:10.1101/cshperspect.a013706

Dias CA et al (2008) Structural modeling and mutational analysis of yeast eukaryotic translation initiation factor $5 \mathrm{~A}$ reveal new critical residues and reinforce its involvement in protein synthesis. FEBS J 275:1874-1888

Firczuk H et al (2013) An in vivo control map for the eukaryotic mRNA translation machinery. Mol Syst Biol 9:635. doi:10.1038/ msb.2012.73

Fraser CS, Berry KE, Hershey JW, Doudna JA (2007) eIF3j is located in the decoding center of the human $40 \mathrm{~S}$ ribosomal subunit. Mol Cell 26:811-819

Gentz PM, Blatch GL, Dorrington RA (2009) Dimerization of the yeast eukaryotic translation initiation factor $5 \mathrm{~A}$ requires hypusine and is RNA dependent. FEBS J 276:695-706. doi:10.1111/j.1742-4658.2008.06817.x

Glick BR, Ganoza MC (1975) Identification of a soluble protein that stimulates peptide bond synthesis. Proc Natl Acad Sci USA 72:4257-4260

Glick BR, Ganoza MC (1976) Characterization and site of action of a soluble protein that stimulates peptide-bond synthesis. Eur J Biochem 71:483-491

Gutierrez E, Shin BS, Woolstenhulme CJ, Kim JR, Saini P, Buskirk AR, Dever TE (2013) eIF5A promotes translation of polyproline motifs. Mol Cell. doi:10.1016/j.molcel.2013.04.021

Jao DLE, Chen KY (2006) Tandem affinity purification revealed the hypusine-dependent binding of eukaryotic initiation factor $5 \mathrm{~A}$ to the translating 80S ribosomal complex. J Cell Biochem 97:583598. doi:10.1002/jcb.20658

Jorgensen P, Nishikawa JL, Breitkreutz BJ, Tyers M (2002) Systematic identification of pathways that couple cell growth and division in yeast. Science 297:395-400. doi:10.1126/ science. 1070850

Kemper WM, Merrick WC, Redfield B, Liu CK, Weissbach H (1976) Purification and properties of rabbit reticulocyte elongation factor-1. Arch Biochem Biophys 174:603-612. doi:10.1016/0003-9861(76)90389-1

Kim KK, Hung LW, Yokota H, Kim R, Kim SH (1998) Crystal structures of eukaryotic translation initiation factor 5A from Methanococcus jannaschii at 1.8 A resolution. Proc Natl Acad Sci USA 95:10419-10424
Koepp DM, Wong DH, Corbett AH, Silver PA (1996) Dynamic localization of the nuclear import receptor and its interactions with transport factors. J Cell Biol 133:1163-1176

Kulak NA, Pichler G, Paron I, Nagaraj N, Mann M (2014) Minimal, encapsulated proteomic-sample processing applied to copynumber estimation in eukaryotic cells. Nat Methods 11:319-324. doi:10.1038/nmeth.2834

Lakowicz JR (2006) Principles of fluorescence spectroscopy, 3rd edn. Springer, New York

Lassak J, Wilson DN, Jung K (2015) Stall no more at polyproline stretches with the translation elongation factors EF-P and IF-5A. Mol Microbiol. doi:10.1111/mmi.13233

Mandal S, Mandal A, Park MH (2015) Depletion of the polyamines spermidine and spermine by overexpression of spermidine/spermine $\mathrm{N}^{1}$-acetyltransferase 1 (SAT1) leads to mitochondria-mediated apoptosis in mammalian cells. Biochem J 468:435-447. doi:10.1042/BJ20150168

Melnikov S et al (2016) Crystal structure of hypusine-containing translation factor eIF5A bound to a rotated eukaryotic ribosome. J Mol Biol. doi:10.1016/j.jmb.2016.05.011

Park MH (2006) The post-translational synthesis of a polyaminederived amino acid, hypusine, in the eukaryotic translation initiation factor 5A (eIF5A). J Biochem (Tokyo) 139:161-169

Park JH, Dias CA, Lee SB, Valentini SR, Sokabe M, Fraser CS, Park MH (2011) Production of active recombinant eIF5A: reconstitution in $E$. coli of eukaryotic hypusine modification of eIF5A by its coexpression with modifying enzymes. Protein Eng Des Sel 24:301-309. doi:10.1093/protein/gzq110

Rodnina MV, Wintermeyer W (2009) Recent mechanistic insights into eukaryotic ribosomes. Curr Opin Cell Biol 21:435-443. doi:10.1016/j.ceb.2009.01.023

Rossi D, Kuroshu R, Zanelli CF, Valentini SR (2014) eIF5A and EF-P: two unique translation factors are now traveling the same road. Wiley Interdiscip Rev RNA 5:209-222. doi:10.1002/wrna.1211

Rossi D et al (2016) Evidence for a negative cooperativity between eIF5A and eEF2 on binding to the ribosome. PLoS One 11:e0154205. doi:10.1371/journal.pone.0154205

Schmidt C et al (2015) Structure of the hypusinylated eukaryotic translation factor eIF-5A bound to the ribosome. Nucleic Acids Res. doi:10.1093/nar/gkv1517

Schnier J, Schwelberger HG, Smit-McBride Z, Kang HA, Hershey JW (1991) Translation initiation factor 5A and its hypusine modification are essential for cell viability in the yeast Saccharomyces cerevisiae. Mol Cell Biol 11:3105-3114

Sokabe M, Fraser CS (2014) Human eukaryotic initiation factor 2 (eIF2)-GTP-Met-tRNAi ternary complex and eIF3 stabilize the 43 S preinitiation complex. J Biol Chem 289:31827-31836. doi:10.1074/jbc.M114.602870

Sun M, Li W, Blomqvist K, Das S, Hashem Y, Dvorin JD, Frank J (2015) Dynamical features of the Plasmodium falciparum ribosome during translation. Nucleic Acids Res 43:10515-10524. doi:10.1093/nar/gkv991

Tong Y, Park I, Hong BS, Nedyalkova L, Tempel W, Park HW (2009) Crystal structure of human eIF5A1: insight into functional similarity of human eIF5A1 and eIF5A2. Proteins 75:1040-1045. doi:10.1002/prot.22378

Valentini SR, Casolari JM, Oliveira CC, Silver PA, McBride AE (2002) Genetic interactions of yeast eukaryotic translation initiation factor 5A (eIF5A) reveal connections to poly(A)-binding protein and protein kinase $\mathrm{C}$ signaling. Genetics 160:393-405

Yang J, Yan R, Roy A, Xu D, Poisson J, Zhang Y (2015) The I-TASSER suite: protein structure and function prediction. Nat Methods 12:7-8. doi:10.1038/nmeth.3213

Zanelli CF et al (2006) eIF5A binds to translational machinery components and affects translation in yeast. Biochem Biophys Res Commun 348:1358-1366 